\title{
Optically Steerable Phased Array Enabling Technology Based on Mesogenic Azobenzene Liquid Crystals for Starlink Towards 6G
}

\author{
Jinfeng $\mathrm{Li}$ \\ Department of Electrical and Electronic Engineering \\ Imperial College London \\ London, UK \\ jinfeng.li@imperial.ac.uk
}

\begin{abstract}
We designed, fabricated, and characterised novel all-optically driven passive phase shifters $(0-\pi)$ at $X$ band based on nematic liquid crystals (GT3) admixed with mesogenic azo dye (CPND7). Thanks to the functional mixture, the developed all-optical phase tuning device enables the removal of voltagedriven networks required by conventional tunable solutions. The embedded light intensity-dependent impedance matching mechanism allows the insertion loss equalisation inherently between phase delaying states, hence removing bulky amplifiers or attenuators for amplitude compensations. The highly scalable planar standalone technology is envisaged to complement the existing electronically steered phased array antenna systems targeting continuous broadband connectivity for SATCOM on the move, and holistically address the future communications demand from various sectors of the economy.
\end{abstract}

Keywords-azo liquid crystals, microwave photonics, mobile broadband, SATCOM, SpaceX, Starlink, 5G, 6G.

\section{INTRODUCTION}

With the maturity of $5 \mathrm{G}$ millimetre-wave technologies, the Internet of Everything [1] is projected to see globally increasing realisation. Within the current framework [2], however, delivering high-speed stable broadband internet remains challenging for areas with unreliable, unaffordable, or unavailable access, hence the need for research and development into 6G communication network and beyond [3], targeting improved quality of service (QoS) that is unbounded by the existing terrestrial infrastructure's limitations [4]. For instance, the basic broadband connectivity solutions available nowadays rely upon maintenance-intensive mechanically stabilised parabolic dish antennas [5], which are heavy and not low-profile. Instead, the envisioned 6G hardware will rely extensively on satellite links via phased array antennas as evidenced by SpaceX's Starlink [6], as well as novel artificial intelligence [7] based architectures.

Among the phased array beam steering technologies, our pioneering work on all-optically addressed microwave phase shifter based on the photoisomerisation of mesogenic azobenzene (azo) liquid crystals (LC) [8] offers a biasnetwork- free solution to partially address the $6 \mathrm{G}$ challenge in terms of the steering performance degradation due to beam squinting [9], power consumption issues (in most tunable dielectrics and magnetic bias waveguide approach) [10][11], and insertion loss unbalance (in existing LC-based variable phase shifters) [11]-[13]. The developed bias-free phaseshifting solution in this work is envisaged to be used in analogue or digital beamforming in a smart and energyefficient way, targeting a moving-parts free, robust, and passive electronically steerable antenna array system with low energy consumptions onboard.

\section{METHODS AND RESUlTS}

\section{A. From Functional Materials to Device Prototype}

The material synthesis and device design were investigated and firstly reported in our pioneering work [8], while this paper steps forward for system-level evaluations towards futuristic applications. Fig. 1 provides a summary from azo LC's photochemically-induced tuning, to phase shifter devices fabrication (see Fig. 2 for the prototyping process from photolithography to capillary filling), towards the envisioned system integration into each phased array flat panel onboard the states-of-the-art Starlink satellite.

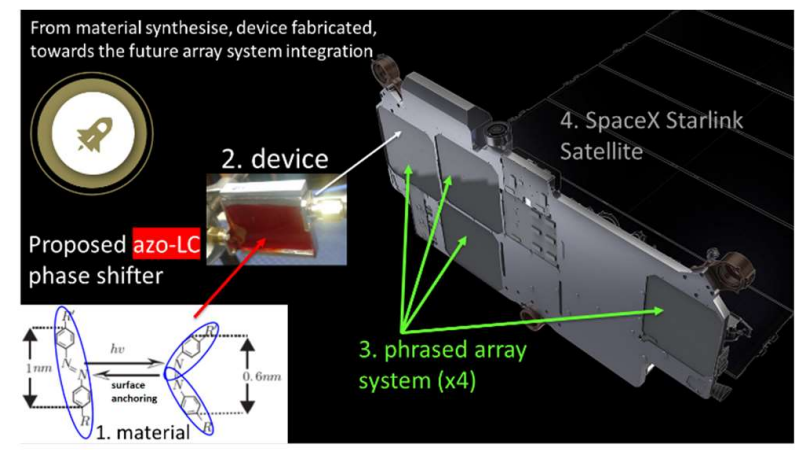

Fig. 1. Progressing from material synthesis to device fabrication, and the envisaged phrased array system integration for a Starlink satellite.

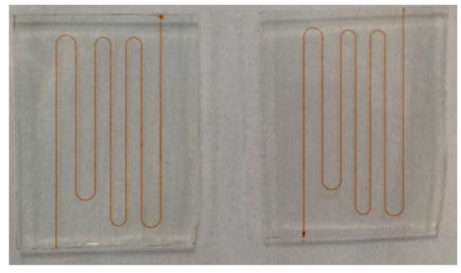

(a)

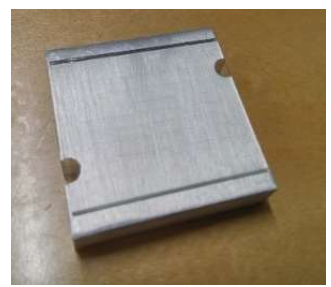

(b)

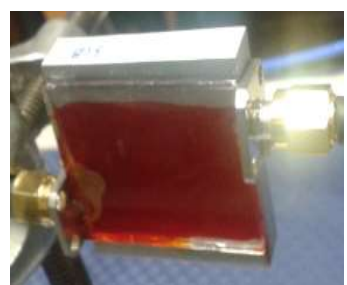

(c)
Fig. 2. Selected illustration of the device prototyping process (a) post-etch microstrip delay line on transparent substrates before stripping photoresist AZ4562; (b) rubbed substrates assembled prior to Azo LC filing; (c) device with Azo LC filled and characterised by VNA via coaxial connectors. 


\section{B. Device Performance Characterisation}

The fabricated azo-LC filled phase shifters are characterised optically (using laser and photodiodes) and electronically (using a vector network analyzer), hence quantifying the light (465 nm wavelength laser) illuminating response concerning the tunable phase delay shown in Fig. 3 (a), and the forward transmission (insertion loss) reported in Fig. 3(b). As demonstrated by Fig. 3(a), continuous phase tuning $(0-\pi)$ is realised for the $\mathrm{X}$ band with enhanced signalto-noise ratios, thus outperforming the conventional digital delay lines in terms of the phase-shifting (beam steering) resolution. More importantly, a nearly flat response in the insertion loss is evidenced in Fig. 3(b) for the device tuned at diverse phase delaying states, which is highly desirable for removing the traditional amplitude-compensating networks [14]. By way of illustration, the measured insertion losses at 8 $\mathrm{GHz}$ are stabilised at around $-2.26 \mathrm{~dB}$, with an average variation of less than $\pm 4.4 \%$ for all phase delaying states. The reasonably small deviation indicates a joint effect of the statistical errors by simulation and the tolerances in fabrication as well, with an implication that the amplitudes could further be homogenised by means of process control $[15][16]$ in the development lifecycle.

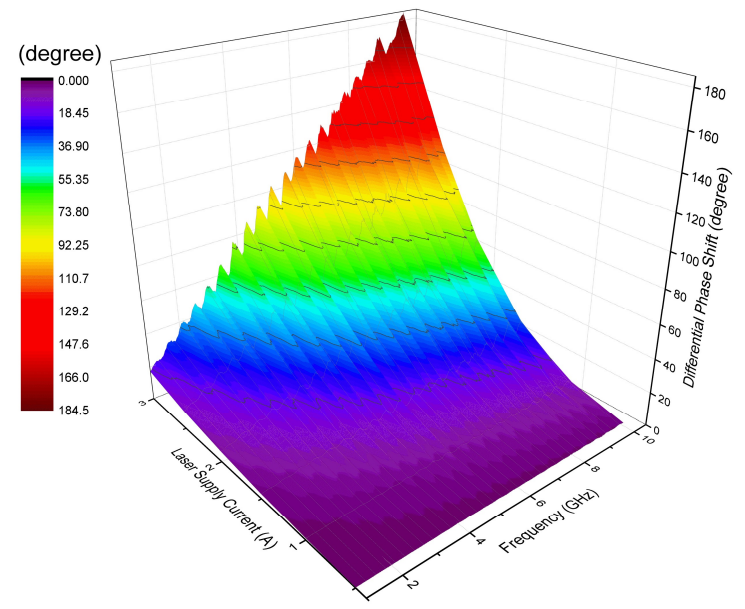

(a)

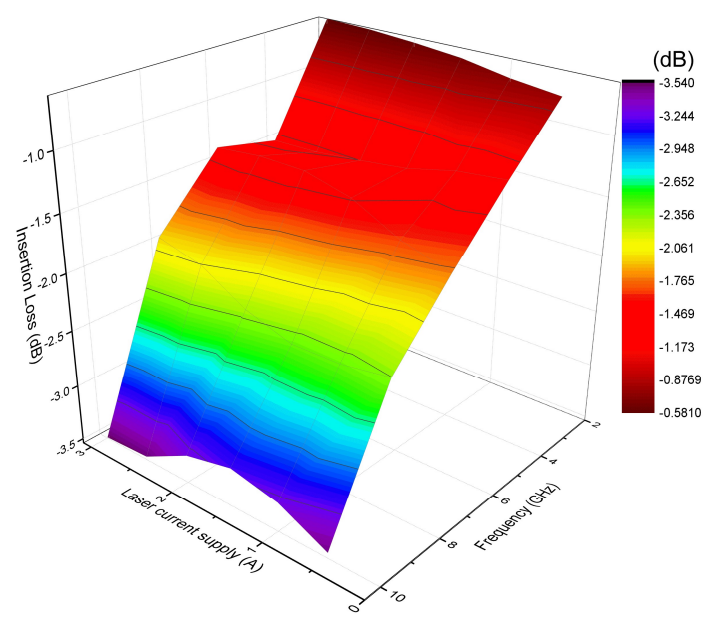

(b)

Fig. 3. Measured (a) differential phase delay (shift); (b) insertion loss of the phase shifter at different frequencies and illumination intensities (different supply currents of the laser).
Based on the validation of results by measurements, the advancement by this work is two-fold. First, as distinguished with the states of the art microwave liquid crystal technology [11][17], the novel azo LC mixture material proposed in this work exploits an alternative tunability-enabling possibility, i.e. all-optical driven for the first time in the microwave passive tunable electronics regime without a bias network [18]. Second, the amplitude balancing of insertion loss exhibits an evolutionary advantage against existing phased array architectures with additional amplitude control networks. However, there remains a gap from evolutionary to revolutionary, due inherently to the reasonably slow tuning response [19][20] of LC materials.

\section{CONCLUSION AND OUTLOOK}

This work pushes the limits of phase-delaying based beam steering with novel optically tunable azo liquid crystals as a functional material to cope with the emerging market demands of enhanced hardware and smart devices with reconfigurability. The agile bias-network free low-profile architecture is scalable to $\mathrm{THz}$ with an enormous amount of throughput for the $6 \mathrm{G}$ framework and bridging the rural fixed broadband gap. Based upon the future leap forward towards phased array system integration, tracking (tuning) speed improvement (lower latency), and industrial implementation to meet the regulatory standard, tremendous use cases could be envisaged, e.g. affordable broadband access onboard trains, streamlined cruise and aircraft operations with ubiquitous connectivity. The embedded redundancy allows failure resistance without the loss of service in vibration and thermal environments on vehicles (aircraft or spacecraft) and satellites. Furthermore, the proposed optically enabled microwave beam steerer can revolutionise the bulky parabolic dish for radio astronomy delving into the origin of the universe and life [21], the origin of the cosmic magnetic field [22], the nature of gravity [23], and the search for extraterrestrial civilization [24].

\section{ACKNOWLEDGMENT}

The author wishes to thank the Centre for Photonic Devices and Sensors (previous Photonics \& Sensors group) in CAPE, University of Cambridge for the training. The author also acknowledges the support from Imperial College London and the University of Southampton.

\section{REFERENCES}

[1] T. Snyder, and G. Byrd, "The Internet of Everything," Computer, vol. 50, no. 6, pp. 8-9, 2017.

[2] A. Karlsson, O. Al-Saadeh, A. Gusarov, R. V. R. Challa, S. Tombaz, and K. W. Sung, "Energy-efficient 5G deployment in rural areas," 2016 IEEE 12th International Conference on Wireless and Mobile Computing, Networking and Communications (WiMob), New York, NY, 2016, pp. 1-7.

[3] M. H. Alsharif, A. H. Kelechi, M. A. Albreem, S. A. Chaudhry, M. S. Zia, and S. Kim, "Sixth generation (6G) wireless networks: Vision, research activities, challenges and potential solutions," Symmetry, vol. 12, no. 4, p. 676, April 2020.

[4] L. U. Khan, I. Yaqoob, M. Imran, Z. Han, and C. S. Hong, "6G Wireless Systems: A Vision, Architectural Elements, and Future Directions," in IEEE Access, vol. 8, pp. 147029-147044, 2020.

[5] T. S. Rappaport, Y. Xing, G. R. MacCartney, A. F. Molisch, E. Mellios, and J. Zhang, "Overview of Millimeter Wave Communications for Fifth-Generation (5G) Wireless Networks-With a Focus on Propagation Models," IEEE Trans. Antennas Propag. vol. 65, no. 12, pp. 6213-6230, December 2017. 
[6] J. Foust, "SpaceX's space-Internet woes: Despite technical glitches, the company plans to launch the first of nearly 12,000 satellites in 2019," IEEE Spectrum, vol. 56, no. 1, pp. 50-51, Janauary 2019.

[7] K. B. Letaief, W. Chen, Y. Shi, J. Zhang, and Y. A. Zhang, "The roadmap to $6 \mathrm{G}$ - AI empowered wireless networks," IEEE Commun. Mag., vol. 57, no. 8, pp. 84-90, August 2019.

[8] J. Li, "All-optically Controlled Microwave Analog Phase Shifter with Insertion Losses Balancing," Engineering Letters, vol. 28, no. 3, pp. 663-667, 2020.

[9] S. K. Garakoui, E. A. M. Klumperink, B. Nauta, and F. E. van Vliet, "Phased-array antenna beam squinting related to frequency dependency of delay circuits," 201141 st European Microwave Conference, Manchester, 2011, pp. 1304-1307.

[10] J. Li, "Structure and Optimisation of Liquid Crystal based Phase Shifter for Millimetre-wave Applications," Apollo, University of Cambridge Repository, doctoral thesis, January 2019. doi: 10.17863/CAM.35704

[11] H. Maune, "Microwave Liquid Crystal Technology," Crystals, vol. 10, 716, August 2020.

[12] J. Li, and D. Chu, "Liquid crystal-based enclosed coplanar waveguide phase shifter for 54-66 GHz applications," Crystals, vol. 9, no. 12, p. 650, December 2019

[13] R. Jakoby, A. Gaebler, and C. Weickhmann, "Microwave Liquid Crystal Enabling Technology for Electronically Steerable Antennas in SATCOM and 5G Millimeter-Wave Systems," Crystals, vol. 10, 514, June 2020.

[14] R. B. Ertel, Zhong Hu, and J. H. Reed, "Antenna array hardware amplitude and phase compensation using baseband antenna array outputs," 1999 IEEE 49th Vehicular Technology Conference (Cat. No.99CH36363), Houston, TX, 1999, vol.3, pp. 1759-1763.

[15] J. Li, "60 GHz Optimised Nickel-free Gold-plated Enclosed Coplanar Waveguide Liquid Crystal Phase Shifter," IEEE MTT-S International Microwave Workshop Series on Advanced Materials and Processes for RF and THz Applications (IEEE MTT-S IMWS-AMP), Suzhou, 2020.
[16] J. Li, "Low-loss tunable dielectrics for millimeter-wave phase shifter: from material modelling to device prototyping," IOP Conference Series: Materials Science and Engineering, vol. 892, p. 012057, 2020.

[17] M. Nickel et al., "Ridge Gap Waveguide Based Liquid Crystal Phase Shifter," in IEEE Access, vol. 8, pp. 77833-77842, 2020.

[18] J. Li, "Bias Tees Integrated Liquid Crystals Inverted Microstrip Phase Shifter for Phased Array Feeds," 21st International Conference on Electronic Packaging Technology (ICEPT), Guangzhou, 2020.

[19] P. Yaghmaee, O. H. Karabey, B. Bates, C. Fumeaux, and R. Jakoby, "Electrically tuned microwave devices using liquid crystal technology," Int. J. Antennas Propagation, vol. 2013, no. 824214, 2013.

[20] L. Cai, H. Xu, J. Li, and D. Chu, "High FoM liquid crystal based microstrip phase shifter for phased array antennas," in 2016 International Symposium on Antennas and Propagation (ISAP), Okinawa, 2016, pp. 402-403.

[21] P. J. Hall, “The Square Kilometre Array: An International Engineering Perspective," Exp. Astron, vol. 17, pp. 5-16, June 2004.

[22] T. G. Arshakian, R. Beck, M. Krause, and D. Sokoloff, "Evolution of magnetic fields in galaxies and future observational tests with the Square Kilometre Array," Astronomy \& Astrophysics, vol. 494, no. 1, pp. 21-32, January 2009.

[23] S. McGee, A. Sesana, and A. Vecchio, "Linking gravitational waves and X-ray phenomena with joint LISA and Athena observations," Nat. Astron, vol. 4, pp. 26-31, Janauary 2020.

[24] K. Tsumura, "Estimating survival probability using the terrestrial extinction history for the search for extraterrestrial life," Sci. Rep., vol. 10, no. 12795, 2020. 